\title{
Hydrogen Transfer between Sulfuric Acid and Hydroxyl Radical in the Gas Phase: Competition among Hydrogen Atom Transfer, Proton-Coupled Electron-Transfer, and Double Proton Transfer
}

\author{
Josep M. Anglada, ${ }^{*, \dagger}$ Santiago Olivella, ${ }^{*, \dagger}$ and Albert Solé
}

Institut d'Investigacions Químiques i Ambientals de Barcelona, CSIC, Jordi Girona 18, 08034Barcelona, Catalonia, Spain and Centre Especial de Recerca en Química Teòrica $i$ Departament de Química Física, Universitat de Barcelona, Martí i Franquès 1, 08028Barcelona, Catalonia, Spain

\section{(Supporting information)}

Table 1S. Cartesian coordinates (in angstrom) for the stationary points of the $\mathrm{H}_{2} \mathrm{SO}_{4}+\mathrm{HO} \bullet$ reaction optimized at the B3LYP/6-311+G(2df,2p) level of theory.

\begin{tabular}{|c|c|c|c|}
\hline \multicolumn{4}{|c|}{$\mathrm{H}_{2} \mathrm{SO}_{4}$} \\
\hline S & 0.000000 & 0.000000 & 0.159680 \\
\hline $\mathrm{O}$ & 0.000000 & 1.260710 & 0.827910 \\
\hline $\mathrm{O}$ & 0.000000 & -1.260710 & 0.827910 \\
\hline $\mathrm{O}$ & -1.243555 & -0.046329 & -0.848038 \\
\hline $\mathrm{O}$ & 1.243555 & 0.046329 & -0.848038 \\
\hline $\mathrm{H}$ & 1.464768 & -0.857126 & -1.116410 \\
\hline $\mathrm{H}$ & -1.464768 & 0.857126 & -1.116410 \\
\hline \multicolumn{4}{|c|}{$\mathrm{H}_{2} \mathrm{O}$} \\
\hline $\mathrm{H}$ & 0.000000 & 0.763192 & -0.466890 \\
\hline $\mathrm{O}$ & 0.000000 & 0.000000 & 0.116723 \\
\hline $\mathrm{H}$ & 0.000000 & -0.763192 & -0.466890 \\
\hline \multicolumn{4}{|c|}{$\mathrm{HSO}_{4}{ }^{\circ}-\mathrm{cis}$} \\
\hline $\mathrm{O}$ & -0.551735 & 0.782307 & 1.137164 \\
\hline S & 0.069017 & 0.071128 & 0.000000 \\
\hline $\mathrm{O}$ & -0.551735 & 0.782307 & -1.137164 \\
\hline $\mathrm{O}$ & 1.496159 & -0.055772 & 0.000000 \\
\hline $\mathrm{O}$ & -0.551735 & -1.395798 & 0.000000 \\
\hline $\mathrm{H}$ & 0.168086 & -2.042398 & 0.000000 \\
\hline \multicolumn{4}{|c|}{$\mathrm{HSO}_{4}{ }^{\circ}-$ trans } \\
\hline $\mathrm{H}$ & 0.400921 & -1.979485 & 0.000000 \\
\hline $\mathrm{O}$ & 0.937097 & -1.172580 & 0.000000 \\
\hline S & 0.000000 & 0.111729 & 0.000000 \\
\hline $\mathrm{O}$ & -0.939208 & -0.013533 & 1.140034 \\
\hline $\mathrm{O}$ & -0.939208 & -0.013533 & -1.140034 \\
\hline
\end{tabular}




\begin{tabular}{|c|c|c|c|}
\hline $\mathrm{O}$ & 0.891204 & 1.223624 & 0.000000 \\
\hline \multicolumn{4}{|c|}{$\mathrm{Cr} 1=\mathrm{Cr} 1$} \\
\hline $\mathrm{S}$ & 0.537543 & -0.049867 & 0.138864 \\
\hline $\mathrm{O}$ & 1.720125 & -0.794872 & 0.423293 \\
\hline $\mathrm{O}$ & -0.307697 & 0.488040 & 1.169605 \\
\hline $\mathrm{O}$ & 0.929602 & 1.210942 & -0.766155 \\
\hline $\mathrm{O}$ & -0.342580 & -0.921103 & -0.840508 \\
\hline $\mathrm{H}$ & -1.290557 & -0.650726 & -0.757907 \\
\hline $\mathrm{H}$ & 1.709080 & 0.989643 & -1.295782 \\
\hline $\mathrm{O}$ & -2.832844 & 0.024298 & -0.092036 \\
\hline $\mathrm{H}$ & -2.352048 & 0.400512 & 0.678260 \\
\hline \multicolumn{4}{|c|}{ TS1 } \\
\hline $\mathrm{O}$ & 0.003360 & -0.149511 & -0.069865 \\
\hline $\mathrm{S}$ & -0.080572 & -0.096490 & 1.438476 \\
\hline $\mathrm{O}$ & 1.311242 & -0.204711 & 1.943913 \\
\hline $\mathrm{O}$ & -0.885603 & 0.972041 & 1.947910 \\
\hline $\mathrm{O}$ & -0.760380 & -1.478224 & 1.873114 \\
\hline $\mathrm{H}$ & -1.695860 & -1.318651 & 2.061164 \\
\hline $\mathrm{H}$ & 1.138459 & -0.461994 & -0.223798 \\
\hline $\mathrm{O}$ & 2.299924 & -0.800709 & 0.024353 \\
\hline $\mathrm{H}$ & 2.839348 & -0.004315 & 0.156109 \\
\hline \multicolumn{4}{|c|}{ TS2 } \\
\hline $\mathrm{O}$ & -0.012033 & -0.087810 & -0.006606 \\
\hline S & -0.010633 & -0.065130 & 1.502714 \\
\hline $\mathrm{O}$ & 1.409488 & -0.197554 & 1.922652 \\
\hline $\mathrm{O}$ & -0.777720 & 0.984365 & 2.101606 \\
\hline $\mathrm{O}$ & -0.700412 & -1.455915 & 1.906440 \\
\hline $\mathrm{H}$ & -1.486637 & -1.261780 & 2.435049 \\
\hline $\mathrm{H}$ & 1.142050 & -0.215447 & -0.242398 \\
\hline $\mathrm{O}$ & 2.366651 & -0.287581 & -0.093377 \\
\hline $\mathrm{H}$ & 2.610910 & -1.202944 & 0.119345 \\
\hline \multicolumn{4}{|c|}{ TS3 } \\
\hline $\mathrm{O}$ & -0.202460 & 0.235473 & 0.049696 \\
\hline S & -0.011884 & 0.162528 & 1.620342 \\
\hline $\mathrm{O}$ & 1.373488 & 0.371361 & 1.934449 \\
\hline $\mathrm{O}$ & -1.060763 & 0.938226 & 2.198462 \\
\hline $\mathrm{O}$ & -0.281002 & -1.383239 & 1.936619 \\
\hline $\mathrm{H}$ & -1.235295 & -1.528394 & 2.009802 \\
\hline $\mathrm{H}$ & 0.645098 & -0.001315 & -0.375238 \\
\hline $\mathrm{O}$ & 2.505813 & -0.313139 & -0.238249 \\
\hline $\mathrm{H}$ & 3.243493 & -0.319075 & 0.394186 \\
\hline \multicolumn{4}{|c|}{ TS4 } \\
\hline S & -0.164895 & 0.075622 & -0.069499 \\
\hline $\mathrm{O}$ & -0.006611 & 0.092009 & 1.351167 \\
\hline $\mathrm{O}$ & 1.102722 & -0.123700 & -0.847670 \\
\hline $\mathrm{O}$ & -1.105987 & -1.160033 & -0.455307 \\
\hline $\mathrm{O}$ & -0.887812 & 1.234666 & -0.679293 \\
\hline $\mathrm{H}$ & -0.162646 & 1.655468 & -1.706381 \\
\hline
\end{tabular}




\begin{tabular}{|c|c|c|c|}
\hline $\mathrm{H}$ & -1.048534 & -1.821270 & 0.248629 \\
\hline $\mathrm{O}$ & 0.716141 & 1.654782 & -2.426510 \\
\hline $\mathrm{H}$ & 1.110219 & 0.779807 & -1.828979 \\
\hline \multicolumn{4}{|c|}{ Cp1 } \\
\hline $\mathrm{O}$ & 0.032783 & 0.206345 & 1.400083 \\
\hline $\mathrm{S}$ & -0.527790 & -0.063124 & 0.110905 \\
\hline $\mathrm{O}$ & 0.421412 & -0.828365 & -0.802234 \\
\hline $\mathrm{O}$ & -1.765090 & -0.809911 & -0.021959 \\
\hline $\mathrm{O}$ & -0.723870 & 1.372837 & -0.549896 \\
\hline $\mathrm{H}$ & -1.165867 & 1.283802 & -1.406889 \\
\hline $\mathrm{H}$ & 2.179306 & 0.192518 & 0.726090 \\
\hline $\mathrm{O}$ & 2.604482 & 0.103284 & -0.138011 \\
\hline $\mathrm{H}$ & 2.873463 & -0.819857 & -0.197539 \\
\hline \multicolumn{4}{|c|}{ Cp2 } \\
\hline $\mathrm{O}$ & 0.044658 & -0.181451 & 1.398548 \\
\hline $\mathrm{S}$ & -0.532124 & -0.091823 & 0.091470 \\
\hline $\mathrm{O}$ & 0.380146 & -0.644125 & -0.994093 \\
\hline $\mathrm{O}$ & -1.813399 & -0.700008 & -0.207879 \\
\hline $\mathrm{O}$ & -0.643497 & 1.480787 & -0.184649 \\
\hline $\mathrm{H}$ & -1.157215 & 1.632469 & -0.991391 \\
\hline $\mathrm{H}$ & 2.207774 & -0.227682 & 0.718521 \\
\hline $\mathrm{O}$ & 2.637220 & -0.059401 & -0.130836 \\
\hline $\mathrm{H}$ & 2.622413 & 0.897969 & -0.239379 \\
\hline
\end{tabular}


Table 2S. Total electronic energy ( $E$, hartree), zero-point vibrational energy $\left(Z P V E, \mathrm{kcal} \mathrm{mol}^{-1}\right)$, enthalpy ( $H$, hartree), Gibbs free-energy ( $G$, hartree), and absolute entropy $(S$, eu) at $298 \mathrm{~K}$ of stationary points for the $\mathrm{H}_{2} \mathrm{SO}_{4}+\mathrm{HO}^{\circ}$ reaction system.

\begin{tabular}{|c|c|c|c|c|c|c|}
\hline \multirow[b]{2}{*}{ Species } & \multicolumn{5}{|c|}{ UB3LYP ${ }^{\mathrm{a}}$} & \multirow{2}{*}{$\frac{\mathrm{UCCSD}(\mathrm{T})^{\mathrm{b}}}{E}$} \\
\hline & $E$ & $Z P V E^{\mathrm{c}}$ & $H$ & $G$ & $S$ & \\
\hline $\mathrm{H}_{2} \mathrm{SO}_{4}$ & -700.41402 & 24.4 & -700.36899 & -700.40277 & 71.1 & -699.42999 \\
\hline $\mathrm{HO}^{\bullet}$ & -75.76506 & 5.3 & -75.75329 & -75.77352 & 42.6 & -75.63771 \\
\hline $\mathrm{H}_{2} \mathrm{O}$ & -76.46256 & 13.4 & -76.43743 & -76.45885 & 45.1 & -76.33214 \\
\hline $\mathrm{HSO}_{4}{ }^{-}$-cis & -699.73561 & 15.9 & -699.70406 & -699.73936 & 74.3 & -698.74384 \\
\hline $\mathrm{HSO}_{4}{ }^{\circ}$-trans & -699.73598 & 16.0 & -699.70440 & -699.73929 & 73.4 & -698.74410 \\
\hline Cr1 & -776.19300 & 31.3 & -776.13453 & -776.17598 & 87.2 & -775.08483 \\
\hline TS1 & -776.18390 & $29.5(-1284)$ & -776.12924 & -776.16856 & 82.7 & -775.06349 \\
\hline TS2 & -776.18315 & $29.4(-1266)$ & -776.12857 & -776.16846 & 84.0 & -775.06292 \\
\hline TS3 & -776.18103 & $28.2(-1685)$ & -776.12802 & -776.16835 & 84.9 & -775.06090 \\
\hline TS4 & -776.17277 & $27.5(-1073)$ & -776.12169 & -776.16023 & 81.1 & -775.063075 \\
\hline Cp1 & -776.20275 & 31.4 & -776.14335 & -776.18626 & 90.3 & -775.08071 \\
\hline Cp2 & -776.20104 & 31.3 & -776.14171 & -776.18526 & 91.7 & -775.07964 \\
\hline
\end{tabular}

${ }^{a}$ Using the 6-311+G(2df,2p) basis set. ${ }^{b}$ Using the cc-pVTZ basis set and the UB3LYP/6-311+G(2df,2p) optimized geometries. ${ }^{\circ}$ The imaginary frequencies $\left(\mathrm{cm}^{-1}\right)$ of the transition structures are given in parentheses. 
Table 3S. Calculated topological properties of the electron charge density at the bond critical points of stationary points for the $\mathrm{H}_{2} \mathrm{SO}_{4}+\mathrm{HO} \bullet$ reaction. ${ }^{a}$

\begin{tabular}{|c|c|c|c|c|c|}
\hline $\mathbf{X}-\mathbf{Y}$ & $\mathbf{r}_{\mathbf{X}}(\mathrm{bohr})$ & $\mathbf{r}_{\mathbf{Y}}(\mathrm{bohr})$ & $\rho\left(\mathrm{e} \mathrm{bohr} r^{-3}\right)$ & $\nabla^{2} \rho\left(\mathrm{e} b o h r^{-5}\right.$ & $\boldsymbol{\varepsilon}$ (hartree bohr ${ }^{-3}$ ) \\
\hline \multicolumn{6}{|c|}{ Stationary point $\mathrm{Cr} 1=\mathrm{Cr} 1$} \\
\hline S4-O3 & 1.0761 & 1.6404 & 0.3245 & 0.6477 & 0.0101 \\
\hline S4-05 & 1.1860 & 1.7987 & 0.2505 & -0.2316 & 0.0901 \\
\hline O5-H6 & 1.5281 & 0.3414 & 0.3347 & -2.5100 & 0.0129 \\
\hline H6-O1 & 1.1503 & 2.2732 & 0.0359 & 0.0984 & 0.0283 \\
\hline O1-H2 & 1.5017 & 0.3557 & 0.3542 & -2.6536 & 0.0356 \\
\hline O3-H2 & 2.4767 & 1.5294 & 0.0187 & 0.0661 & 0.1497 \\
\hline S4-07 & 1.0693 & 1.6260 & 0.3312 & 0.7548 & 0.0122 \\
\hline S4-08 & 1.2099 & 1.8168 & 0.2396 & -0.2913 & 0.0810 \\
\hline О8-H9 & 1.4807 & 0.3487 & 0.3616 & -2.7236 & 0.0149 \\
\hline Ring & & & 0.0120 & 0.0505 & \\
\hline
\end{tabular}

$\begin{array}{cc}\text { S4-O3 } & 1.1062 \\ \text { S4-O5 } & 1.1262 \\ \text { O3-O1 } & 2.1407 \\ \text { O5-H6 } & 1.7105 \\ \text { H6-O1 } & 0.5732 \\ \text { O1-H2 } & 1.4785 \\ \text { S4-O7 } & 1.0722 \\ \text { S4-O8 } & 1.2082 \\ \text { O8-H9 } & 1.4820 \\ \text { Ring } & \end{array}$

Stationary point TS1

$\begin{array}{llcl}1.6995 & 0.3007 & 0.2463 & 0.0621 \\ 1.7305 & 0.2859 & 0.0667 & 0.0738 \\ 2.0952 & 0.0394 & 0.1684 & 0.0813 \\ 0.5336 & 0.1822 & -0.4651 & 0.0061 \\ 1.7609 & 0.1627 & -0.2771 & 0.0022 \\ 0.3562 & 0.3647 & -2.7278 & 0.0018 \\ 1.6330 & 0.3273 & 0.7199 & 0.0124 \\ 1.8164 & 0.2400 & -0.2888 & 0.0897 \\ 0.3463 & 0.3613 & -2.7303 & 0.0156 \\ & 0.0281 & 0.1381 & \end{array}$

$\begin{array}{cc}\text { S4-O3 } & 1.1081 \\ \text { S4-O5 } & 1.1245 \\ \text { O3-O1 } & 2.1391 \\ \text { O5-H6 } & 1.7099 \\ \text { H6-O1 } & 0.5738 \\ \text { O1-H2 } & 1.4791 \\ \text { S4-O7 } & 1.0721 \\ \text { S4-O8 } & 1.2130 \\ \text { O8-H9 } & 1.4827 \\ \text { Ring } & \end{array}$

Stationary point TS2

$\begin{array}{lccc}1.7017 & 0.2996 & 0.2268 & 0.0695 \\ 1.7281 & 0.2868 & 0.0775 & 0.0751 \\ 2.0847 & 0.0397 & 0.1683 & 0.0849 \\ 0.5294 & 0.1836 & -0.4869 & 0.0075 \\ 1.7617 & 0.1612 & -0.2656 & 0.0035 \\ 0.3558 & 0.3646 & -2.7289 & 0.0189 \\ 1.6326 & 0.3275 & 0.7206 & 0.0011 \\ 1.8190 & 0.2381 & -0.2992 & 0.0879 \\ 0.3449 & 0.3617 & -2.7407 & 0.0166 \\ & 0.0281 & 0.1375 & \end{array}$

$\begin{array}{lc}\text { S4-O3 } & 1.0736 \\ \text { S4-O5 } & 1.1666 \\ \text { O3-H2 } & 2.7326 \\ \text { O5-H6 } & 1.6620 \\ \text { H6-O1 } & 0.57231 \\ \text { O1-H2 } & 1.4925 \\ \text { S4-O7 } & 1.0745\end{array}$

Stationary point TS3

$\begin{array}{llcl}1.6360 & 0.3266 & 0.6855 & 0.0096 \\ 1.7822 & 0.2583 & -0.1333 & 0.0599 \\ 1.9108 & 0.0115 & 0.0433 & 2.0767 \\ 0.5248 & 0.1961 & -0.5431 & 0.0286 \\ 1.7046 & 0.1721 & -0.2938 & 0.0193 \\ 0.3538 & 0.3584 & -2.6955 & 0.0237 \\ 1.6380 & 0.3256 & 0.6758 & 0.0153\end{array}$




$\begin{array}{cccccc}\text { S4-O8 } & 1.2061 & 1.8158 & 0.2401 & -0.2794 & 0.0776 \\ \text { O8-H9 } & 1.4808 & 0.3486 & 0.3616 & -2.7243 & 0.0145 \\ \text { Ring } & & & 0.0115 & 0.0467 & \end{array}$

$\begin{array}{cccccc}\text { S4-O3 } & 1.1186 & 1.7175 & 0.2906 & 0.1419 & 0.0510 \\ \text { S4-O5 } & 1.1148 & 1.7123 & 0.2931 & 0.1708 & 0.0467 \\ \text { O3-H2 } & 1.8359 & 0.6873 & 0.1209 & 0.0143 & 0.0052 \\ \text { O5-H6 } & 1.8285 & 0.6785 & 0.1237 & 0.0005 & 0.0045 \\ \text { H6-O1 } & 0.4668 & 1.6801 & 0.2200 & -0.976 & 0.0297 \\ \text { O1-H2 } & 1.6755 & 0.4608 & 0.2237 & -1.0281 & 0.0295 \\ \text { S4-O7 } & 1.0711 & 1.6303 & 0.3289 & 0.7302 & 0.0158 \\ \text { S4-O8 } & 1.2084 & 1.8169 & 0.2397 & -2.9146 & 0.0774 \\ \text { O8-H9 } & 1.4817 & 0.3467 & 0.3615 & -2.7307 & 0.0158 \\ \text { Ring } & & & 0.0260 & 0.1365 & \end{array}$

$\begin{array}{cccccc}\text { S4-O3 } & 1.1332 & 1.7459 & 0.2784 & 0.0514 & 0.0644 \\ \text { S4-O5 } & 1.0719 & 1.6300 & 0.3281 & 0.7079 & 0.0055 \\ \text { O3-O1 } & 2.2736 & 2.3845 & 0.0239 & 0.0922 & 0.0201 \\ \text { O5-H6 } & 2.6421 & 1.6627 & 0.0136 & 0.0512 & 0.5829 \\ \text { H6-O1 } & 0.3485 & 1.4792 & 0.3655 & -2.7513 & 0.0134 \\ \text { O1-H2 } & 1.4654 & 0.3551 & 0.3704 & -2.7587 & 0.0137 \\ \text { S4-O7 } & 1.0835 & 1.6593 & 0.3158 & 0.5564 & 0.0225 \\ \text { S4-O8 } & 1.1986 & 1.8128 & 0.2413 & -0.2434 & 0.0837 \\ \text { O8-H9 } & 1.4823 & 0.3478 & 0.3611 & -2.7245 & 0.0131 \\ \text { Ring } & & & 0.0123 & 0.0486 & \end{array}$

$\begin{array}{cccccc}\text { S4-O3 } & 1.1321 & 1.7441 & 0.2800 & 0.0515 & 0.0689 \\ \text { S4-O5 } & 1.0718 & 1.6330 & 0.3277 & 0.7154 & 0.0053 \\ \text { O3-O1 } & 2.2892 & 2.4104 & 0.0226 & 0.0855 & 0.0611 \\ \text { O5-H6 } & 2.6510 & 1.6930 & 0.0133 & 0.0494 & 0.5820 \\ \text { H6-O1 } & 0.3490 & 1.4770 & 0.3664 & -2.7550 & 0.0145 \\ \text { O1-H2 } & 1.4620 & 0.3590 & 0.3706 & -2.7403 & 0.0150 \\ \text { S4-O7 } & 1.0826 & 1.6568 & 0.3170 & 0.5673 & 0.0232 \\ \text { S4-O8 } & 1.2080 & 1.8179 & 0.2383 & -0.2732 & 0.0837 \\ \text { O8-H9 } & 1.4804 & 0.3496 & 0.3616 & -2.7194 & 0.0137 \\ \text { Ring } & & & 0.0120 & 0.0468 & \end{array}$

${ }^{a}$ Determined from Bader topological analysis of the electron charge density calculated at the B3LYP/6-311+G(2df,2p) level. Atom numbering refers to Figure 3 
Table 4S. Harmonic frequencies ${ }^{\mathrm{a}}\left(\mathrm{in}^{-1}\right.$ ) and IR intensities (in $\mathrm{km} \mathrm{mol}^{-1}$ ) calculated at the UB3LYP/6-311+G(2df,2p) level for $\mathrm{H}_{2} \mathrm{SO}_{4}, \mathrm{HO}^{*}$, and the hydrogen-bonded $\mathrm{Cr} \mathbf{1}$ complex.

\begin{tabular}{|c|c|c|c|c|}
\hline species & mode & approx assgnmt & freq $^{b}$ & IR intens ${ }^{c}$ \\
\hline \multirow[t]{15}{*}{$\mathrm{H}_{2} \mathrm{SO}_{4}$} & $v_{1}$ & OH sym stretch & 3628 & 50 \\
\hline & $v_{2}$ & $\mathrm{OH}$ asym stretch & 3624 & 207 \\
\hline & $v_{3}$ & $\mathrm{O}=\mathrm{S}=\mathrm{O}$ asym stretch & 1392 & 304 \\
\hline & $v_{4}$ & $\mathrm{O}=\mathrm{S}=\mathrm{O}$ sym stretch & 1154 & 167 \\
\hline & $v_{5}$ & SOH sym bend & 1120 & 84 \\
\hline & $v_{6}$ & SOH asym bend & 1106 & 78 \\
\hline & $v_{7}$ & $\mathrm{~S}(\mathrm{OH})_{2}$ asym stretch & 818 & 342 \\
\hline & $v_{8}$ & $\mathrm{~S}(\mathrm{OH})_{2}$ sym stretch & 768 & 113 \\
\hline & $v_{9}$ & OSO wag & 519 & 24 \\
\hline & $v_{10}$ & $\mathrm{O}=\mathrm{S}=\mathrm{O}$ bend & 510 & 3 \\
\hline & $v_{11}$ & $(\mathrm{H}) \mathrm{OSO}(\mathrm{H})$ wag & 467 & 41 \\
\hline & $v_{12}$ & $(\mathrm{H}) \mathrm{OSO}(\mathrm{H})$ bend & 412 & 16 \\
\hline & $v_{13}$ & $\mathrm{SO}(\mathrm{H})$ twist & 350 & 2 \\
\hline & $v_{14}$ & symm SOH torsion & 309 & 60 \\
\hline & $v_{15}$ & asym $\mathrm{SOH}$ torsion & 235 & 101 \\
\hline $\mathrm{HO}^{\bullet}$ & $v_{1}$ & $\mathrm{OH}$ stretch & 3571 & 15 \\
\hline \multirow[t]{12}{*}{ Cr1 } & $v_{1}$ & O8H9 stretch & $3627(-1)$ & $129(2.6)$ \\
\hline & $v_{2}$ & $\mathrm{O} 1 \mathrm{H} 2$ stretch & $3453(-118)$ & $155(10.3)$ \\
\hline & $v_{3}$ & O5H6 stretch & $3230(-394)$ & $784(3.8)$ \\
\hline & $v_{4}$ & $\mathrm{O}=\mathrm{S}=\mathrm{O}$ asym stretch & $1383(-9)$ & $312(1.0)$ \\
\hline & $v_{5}$ & $\mathrm{O}=\mathrm{S}=\mathrm{O}$ sym stretch & $1141(-13)$ & $124(0.7)$ \\
\hline & $v_{6}$ & SO5H6 bend & $1245(+125)$ & $83(1.0)$ \\
\hline & $v_{7}$ & SO8H9 bend & $1109(+3)$ & $117(1.5)$ \\
\hline & $v_{8}$ & $\mathrm{~S}(\mathrm{OH})_{2}$ asym stretch & $849(+31)$ & $317(0.9)$ \\
\hline & $v_{9}$ & $\mathrm{~S}(\mathrm{OH})_{2}$ sym stretch & $780(+12)$ & $125(1.1)$ \\
\hline & $v_{10}$ & $(\mathrm{SO} 5 \mathrm{H} 6+\mathrm{O} 1 \mathrm{H} 2)$ rock & 588 & 121 \\
\hline & $v_{11}$ & OSO wag & $527(+8)$ & 31 (1.3) \\
\hline & $v_{12}$ & $\mathrm{O}=\mathrm{S}=\mathrm{O}$ bend & $517(+7)$ & $34(0.9)$ \\
\hline
\end{tabular}




$\begin{array}{lllr}v_{13} & (\mathrm{SO} 5 \mathrm{H} 6+\mathrm{O} 1 \mathrm{H} 2) \text { rock } & 508 & 218 \\ v_{14} & (\mathrm{H}) \mathrm{OSO}(\mathrm{H}) \text { wag } & 474(7) & 40(1.0) \\ v_{15} & (\mathrm{H}) \mathrm{OSO}(\mathrm{H}) \text { bend } & 401(-11) & 45(2.8) \\ v_{16} & \mathrm{SO}(\mathrm{H}) \text { twist } & 351(+1) & 3(1.3) \\ v_{17} & \text { O1H2 twist } & 266 & 111 \\ v_{18} & \text { SO8H9 torsion } & 249(-60) & 82(1.4) \\ v_{19} & \text { intermol }(\mathrm{OH}+\text { O5H6) stretch } & 181 & 20 \\ v_{20} & \text { intermol }(\mathrm{OH}+\text { O5H6) bend } & 139 & 36 \\ v_{21} & \text { intermol }(\mathrm{OH}+\text { O5H6) torsion } & 44 & 2\end{array}$

${ }^{\text {a }}$ Scaled by the factor $0.9614 .{ }^{b}$ Frequency shifts relative to the complex components are given in parentheses. 'Intensity ratio relative to the complex components are given in parentheses. 

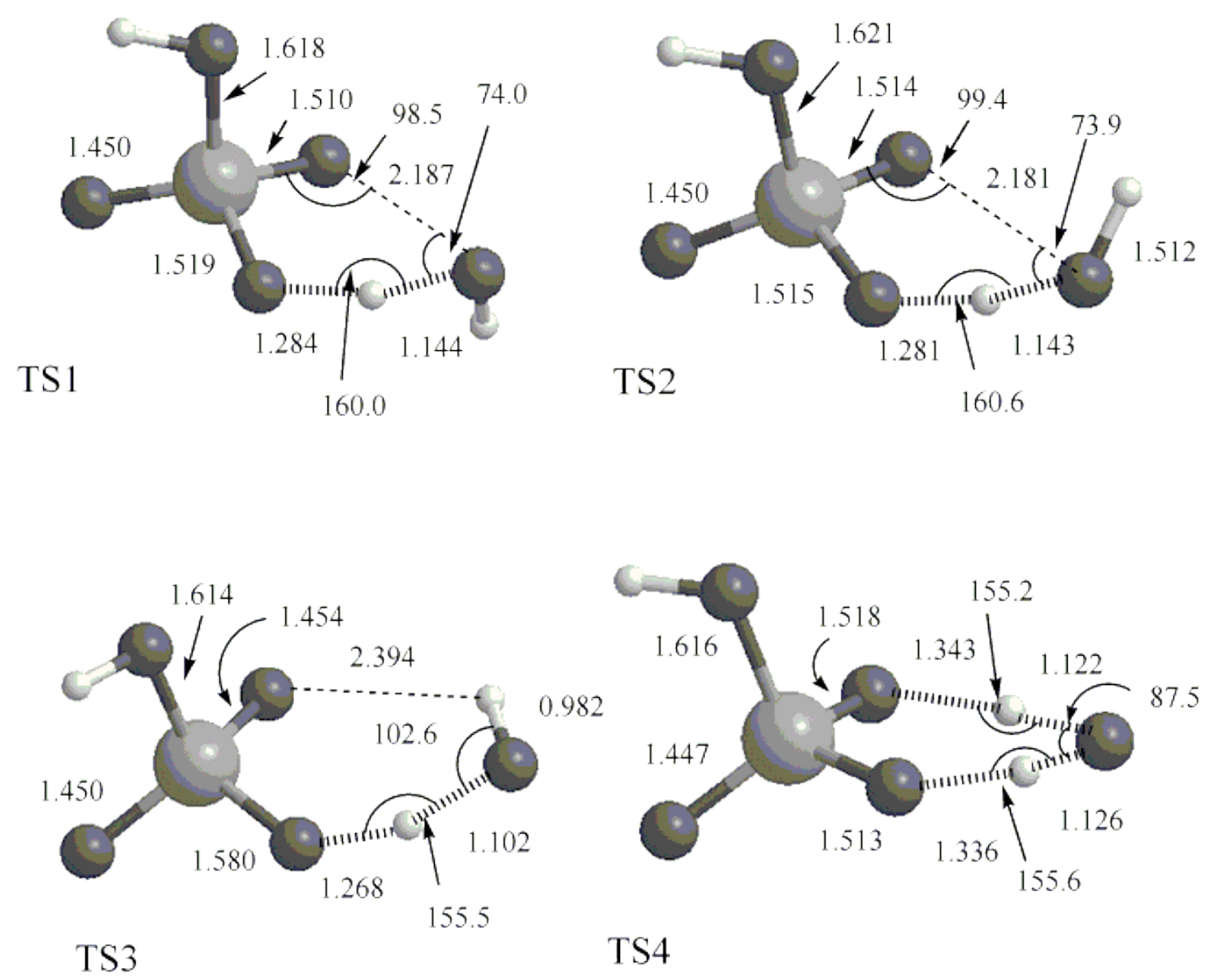

Figure S1 